\title{
Methacarn as a whole brain fixative for gene and protein expression analyses of specific brain regions in rats
}

\author{
Hirotoshi Akane1, Fumiyo Saito², Hidenori Yamanaka², Ayako Shiraki ${ }^{1,3}$, \\ Nobuya Imatanaka², Yumi Akahori², Reiko Morita1,3, Kunitoshi Mitsumori' \\ and Makoto Shibutani ${ }^{1}$ \\ ${ }^{1}$ Laboratory of Veterinary Pathology, Tokyo University of Agriculture and Technology, \\ 3-5-8 Saiwai-cho, Fuchu-shi, Tokyo 183-8509, Japan \\ ${ }^{2}$ Chemicals Evaluation and Research Institute, Japan, 1-4-25 Koraku, Bunkyo-ku, Tokyo 112-0004, Japan \\ ${ }^{3}$ Pathogenetic Veterinary Science, United Graduate School of Veterinary Sciences, Gifu University, \\ 1-1 Yanagido, Gifu-shi, Gifu 501-1193, Japan
}

(Received February 16, 2013; Accepted March 22, 2013)

\begin{abstract}
For molecular analysis in anatomically-specific brain regions for rodent studies, it is necessary to establish a fast and accurate procedure for tissue sampling to achieve high integrity and expression fidelity of extracted molecules. The present study was performed to examine suitability of whole brain fixation with methacarn and subsequent tissue sampling using punch-biopsy devices for gene expression analysis in rats. After fixation, each specific region, i.e., hippocampal dentate gyrus, corpus callosum, cingulate cortex or cerebellar vermis was collected, and the integrity and variability of expression data of extracted total RNAs and polypeptides were examined. Methacarn fixation, acetone fixation, and unfixed tissues were compared. Methacarn fixation resulted in high integrity of total RNAs sufficient for conducting global expression analysis and superior in terms of uniformity in the integrity among brain regions to that of acetone fixation. Extracted polypeptide after methacarn fixation revealed similar integrity to that without fixation or with acetone fixation. Methacarn fixation resulted in lower mRNA expression variability between samples than acetone fixation in microarray analysis. The fidelity of polypeptide expression was mostly equivalent between methacarn and acetone fixation in 2-dimensional differential in-gel electrophoresis, although the expression levels of a small number of polypeptides from acetonefixed tissues were affected. These results suggest that whole brain fixation with methacarn retains advantages for global analyses of mRNAs and polypeptides in rodent studies.
\end{abstract}

Key words: Methacarn, Microarray analysis, Proteome analysis, Tissue sampling, Specific brain region

\section{INTRODUCTION}

The central nervous system has an anatomically elaborate architecture with region-specific differences in the distribution of neuronal and glial cell populations. For molecular biological analyses in each anatomically-specific region, each region must be sampled separately and the accurate sampling may be achieved with utilization of appropriate tissue fixation. On the other hand, as macromolecules (i.e. DNA, RNA and polypeptides) degrade gradually following autopsy, the time required to complete manual sampling of multiple specific brain regions in small animal experiments can result in a reduction in the quality of molecular biological analyses. Recent studies have applied microdissection techniques to analyze genes and proteins in anatomically-specific tissue regions (Böhm et al., 2005; Ornstein et al., 2000; Woo et al., 2009). However, microdissection techniques are generally laborious and a low throughput method of tissue sampling. Applying whole brain fixation may be necessary to preserve high integrity of macromolecules and allow delayed processing of removed brains for rapid sampling of anatomically-specific brain regions following autopsy in animal experiments. To achieve consistent tissue sampling of specific brain regions within an appropriate operating time, manual dissection methods allow high

Correspondence: Makoto Shibutani (E-mail: mshibuta@cc.tuat.ac.jp) 
throughput and use of fixed brain samples may be preferable for this purpose.

Methacarn is a protein-precipitating and non-crosslinking organic solvent fixative (Mitchell et al., 1985; Puchtler et al., 1970), and fixation with this solution enables preservation of tissue morphology for histopathological assessment in paraffin-embedded tissues (Delfour et al., 2006; Srinivasan et al., 2002). Methacarn generally gives superior immunohistochemical results over aldehyde-based cross-linking fixatives (Banks, 1979; Orstavik et al., 1981; Rognum et al., 1980) because antigenicity is usually maintained (Mitchell et al., 1985). We previously found that methacarn fixation yields high quality DNA, RNA and protein even in paraffin-embedded sections for analyses of genomic DNA, expression microarrays, real-time RT-PCR and western blotting (Shibutani et al., 2000; Shibutani and Uneyama, 2002; Takagi et al., 2004; Uneyama et al., 2002).

For global assessment of genes and proteins in anatomically-specific brain regions in animal studies using rodents, both high integrity of extracted molecules and minimal inter-animal variability in the expression of extracted macromolecules are essential. Therefore, establishment of a procedure for quick and accurate tissue sampling and processing which can overcome these hurdles is necessary. For this purpose, development of a method of brain fixation that preserves molecular integrity in the tissue is necessary.

Approaches of toxicogenomics, transcriptomics, and proteomics have recently been applied in many organs in toxicity studies using rodents (Heijne et al., 2005). The present study was performed to examine suitability of methacarn fixation for global expression analysis of mRNAs and proteins in anatomically-specific brain regions for a scheme of regular rodent toxicity studies. We applied a simple and rapid tissue sampling method using punch-biopsy devices after whole brain fixation without paraffin-embedding. We examined the integrity of extracted molecules and fidelity of expression data with regard to the total RNAs and polypeptides extracted from each portion of the specific brain region in rats. We also compared methacarn fixation with fixation in icecold acetone, a method previously used for molecular analysis (Goldsworthy et al., 1999; Yan et al., 2010). We used brains at the time point of the cessation of chemical exposure in a developmental neurotoxicity study on postnatal day (PND) 21 and in a 28-day repeated oral toxicity study on PND 63.

\section{MATERIALS AND METHODS}

\section{Animals and experimental conditions}

Seven-week-old male Crj:CD(SD)IGS rats and pregnant female rats of the same strain at gestational day 2 were purchased from Charles River Japan (Kanagawa, Japan) and maintained in an air-conditioned animal room (temperature: $23 \pm 2^{\circ} \mathrm{C}$, relative humidity: $55 \pm 15 \%$ ) with a 12-hr light/dark cycle. Animals were provided pelleted basal diet (Oriental Yeast Co., Ltd., Tokyo, Japan) and tap water ad libitum.

Two individual animal experiments were carried out. In Experiment 1, 20 male rats were orally administered distilled water ( $5 \mathrm{ml} / \mathrm{kg}$ body weight) repeatedly for 7 days after one-week acclimation. They were randomly divided into 3 groups just before autopsy: unfixed $(\mathrm{N}=4)$, methacarn-fixed or acetone-fixed tissues $(\mathrm{N}=8$ for each fixation). In Experiment 2, 10 pregnant rats were maintained without any treatment. After delivery, offspring at PND 4 were culled to preserve eight pups per litter keeping as many male animals as possible. On PND 21, male offspring were randomly divided into 3 groups, i.e., 8 for unfixed tissue samples and 16 each for fixed tissue samples. Remaining offspring were used for other experimental purposes. Male animals in Experiment 1 were housed with four animals per cage and pregnant rats in Experiment 2 were housed individually with their offspring in plastic cages with wood chip bedding. All animals used in the present study were killed by exsanguination from the abdominal aorta under anesthesia. All procedures of this study were conducted in compliance with the Guidelines for Proper Conduct of Animal Experiments (Science Council of Japan, June 1, 2006) and according to the protocol approved by the Animal Care and Use Committee of the Tokyo University of Agriculture and Technology. All efforts were made to minimize animal suffering.

\section{Tissue fixation and sampling of specific brain regions}

Methacarn solution consisting of $60 \%(\mathrm{v} / \mathrm{v})$ absolute methanol, 30\% chloroform, and 10\% glacial acetic acid was freshly prepared before fixation (Shibutani et al., 2000). Methacarn solution and acetone were stored at $4^{\circ} \mathrm{C}$ until use. At autopsy, whole brains were removed and fixed in the appropriate fixative for $5 \mathrm{hr}$ at $4^{\circ} \mathrm{C}$ with agitation. Fixed brains were dehydrated three times for $1 \mathrm{hr}$ in fresh $99.5 \%$ ethanol and stored overnight at $4{ }^{\circ} \mathrm{C}$ with agitation until each specific brain region was sampled. For comparison of the integrity of extracted total RNA and polypeptides as well as the fidelity in the polypeptide expression from fixed tissues, unfixed whole 
Methacarn for global expression analysis

brains were immersed in ice-cold saline and sampled immediately.

For fixed brains, two $2 \mathrm{~mm}$-thick coronal cerebral slices and two $2 \mathrm{~mm}$-thick sagittal cerebellar slices were prepared. Coronal slices were prepared by cutting laterally at the approximate positions of $-0.8 \mathrm{~mm},-2.8 \mathrm{~mm}$ and $-4.8 \mathrm{~mm}$ from the bregma using the brain-matrix cast (Muromachi Kikai Co., Ltd., Tokyo, Japan). As target regions for this validation, we selected hippocampal dentate gyrus, corpus callosum, cingulate cortex and cerebellar vermis for analysis. Hippocampal dentate gyrus and cingulate cortex were punched from the posterior cerebral slice. The corpus callosum and cerebellar vermis were punched from two cerebral slices and two cerebellar slices, respectively. Sampling was made using punchbiopsy devices with a pore size of $1 \mathrm{~mm}$ (Kai Industries Co., Ltd., Gifu, Japan). Cingulate cortex and cerebellar vermis from unfixed tissues were punched from similar portions of the cerebral and cerebellar slices using 3 mm-sized punch-biopsy devices. Punched tissue samples from fixed brains were immersed in $99.5 \%$ ethanol and stored at $-80^{\circ} \mathrm{C}$. For unfixed tissues, the samples for RNA analysis were immersed in RNAlater ${ }^{\mathrm{TM}}$ (Ambion, Inc., Austin, TX, USA) for $24 \mathrm{hr}$ at $4^{\circ} \mathrm{C}$ and those for polypeptide analysis were quickly frozen in liquid nitrogen for storage at $-80^{\circ} \mathrm{C}$ until macromolecule extraction.

\section{RNA preparation}

Isolation of total RNA from tissue samples was performed using QIAzol (Qiagen, Valencia, CA, USA) together with miRNeasy kit (Qiagen) according to the manufacturer's protocol. Nucleic acid concentration was determined by measurement of optical density at $260 \mathrm{~nm}$ (NanoDrop ND-1000, Thermo Scientific, Wilmington, DE, USA). The RNA integrity was evaluated in 4 animals at PND 63 (Experiment 1) and 8 animals at PND 21 (Experiment 2) and an RNA Integrity Number (RIN) was generated using an Agilent 2100 Bioanalyzer and its accompanying software (Agilent Technologies, Inc., Santa Clara, CA, USA).

\section{Gene expression microarray analysis}

Gene expression analysis was conducted using Agilent Rat Oligo arrays with approximately 60,000 probes for known genes and expressed sequence tags (Agilent Technologies, Inc.) in each brain region of 3 animals each at both PND 63 (Experiment 1) and PND 21 (Experiment 2). For sample preparation and array processing, the Agilent protocol "One-Color Microarray-Based Gene Expression Analysis" was used. Briefly, the recommended volume of control RNAs (Agilent One-Color RNA Spike-In Kit) was added to $100 \mathrm{ng}$ of total RNA. Thereafter, Cy3-labeled cRNA was produced using the Agilent Low Input Quick Amp Labeling (one-color), purified with the RNeasy mini kit (Qiagen), fragmented using the in situ Hybridization Kit (Agilent Technologies, Inc.), and subjected to hybridization by incubation in a hybridization oven (Agilent Technologies, Inc.). Hybridized slides were scanned (G4900DA scanner, Agilent Technologies, Inc.), and data were obtained using Agilent Feature Extraction software (version 11.7.1.1), with defaults for all parameters.

\section{Microarray data normalization and analysis}

Microarray data analyses were carried out using GeneSpring GX (version 11.5.1) software (Agilent Technologies, Inc.). The data were subjected to normalization by setting measurements less than 0.01 to 0.01 and by per-chip and per-gene normalization using GeneSpring normalization algorithms (Agilent Technologies, Inc.).

\section{Polypeptide preparation}

The brain tissue samples in each brain region of 4 animals at PND 63 in Experiment 1 were homogenized in lysis buffer (4\% (w/v) 3-[(3-cholamidopropyl) dimethylammonio] propanesulfonate, $2 \mathrm{M}$ thiourea, $8 \mathrm{M}$ urea, 10 $\mathrm{mM}$ Tris-HCl; $\mathrm{pH} 8.8$ ) and sonicated. Protein concentration was determined using the Bradford method after removing pellets by centrifugation.

\section{Sodium dodecyl sulfate-polyacrylamide gel electrophoresis (SDS-PAGE)}

One-dimensional SDS-PAGE was performed on the Hoefer Mighty Small gel system (GE Healthcare, Little Chalfont, UK). The $5 \mu \mathrm{g}$ of proteins extracted from each brain sample were labeled with $20 \mathrm{pmol}$ of $N$-hydroxysuccinimide (NHS) ester-derivatives of $\mathrm{Cy} 5$, and the labeled protein samples were added to $5 \mu$ of SDS buffer $(2 \%$ SDS, $20 \%$ glycerol, $0.01 \%$ bromphenol blue, and $50 \mathrm{mM}$ Tris-HCl, 0.1 M dithiothreitol, $\mathrm{pH} 6.8$ ), boiled at $95^{\circ} \mathrm{C}$ for $3 \mathrm{~min}$ and applied to $15-25 \%$ gradient acrylamide gel (Cosmo Bio Co., Ltd., Tokyo, Japan). Gels were scanned directly with Typhoon (GE Healthcare). Gel image analysis was performed with Image Quant TL v2005 (GE Healthcare).

\section{Two-dimensional differential in-gel electrophoresis (2D-DIGE) analysis}

The protein lysates as prepared for SDS-PAGE analysis were also labeled with NHS ester-derivatives of $\mathrm{Cy} 2$, Cy3 and Cy5 (GE Healthcare) according to the manufacturer's protocol. Differentially labeled samples were 


\section{H. Akane et al.}

reduced with $65 \mathrm{mM}$ DTT and mixed together with Pharmalytes $\mathrm{pH}$ 3-10 (GE Healthcare). Immobilized $\mathrm{pH}$ gradient strips $(\mathrm{pH} 3-10$; Length $=24 \mathrm{~cm})$ were rehydrated and mixed samples were applied to strips. Isoelectric focusing was performed using a Multiphor II (GE Healthcare). 2D-gels were run in Ettan DALT twelve (GE Healthcare) and were scanned with a Typhoon 9400 (GE Healthcare). The images were analyzed for protein profile by Decyder $^{\circledR}$ software Ver7.5 (GE Healthcare).

\section{Image analysis}

The differential in-gel analysis (DIA) of DeCyder ${ }^{\circledR}$ was applied to merge the $\mathrm{Cy} 2, \mathrm{Cy} 3$ and $\mathrm{Cy} 5$ images for each gel, and then to detect spot boundaries for the calculation of normalized spot volumes/protein abundance. Differences in the protein abundance were then calculated between samples run on the same gel. The biological variation analysis of DeCyder ${ }^{\circledR}$ was applied to match all pair wise image comparisons from DIA for a comparative cross-gel statistical analysis.

\section{Mass spectrometric (MS) analysis}

Gel electrophoresis for MS analysis was performed using $600 \mu \mathrm{g}$ of pooled lysate prepared as described above. The resultant gel was stained with Sypro ${ }^{\circledR}$ Ruby, then spots of interest were excised from 2D-gels using an automated spot picker (GE Healthcare). The recovered gel pieces were incubated with $12.5 \mathrm{ng} / \mu \mathrm{l}$ trypsin (Promega Corporation, Madison, WI, USA) and generated peptides were eluted and dissolved in $0.1 \% \mathrm{v} / \mathrm{v}$ formic acid.

MS analysis was carried out by LC-MS/MS. NanoUPLC (Waters, Milford, MA, USA) was coupled with the Q-TOF micro mass spectrometer (Micromass, Manchester, UK). Instrument operation, data acquisition and analysis were performed using MassLynx software (Micromass). The eluted peptide was analyzed by tandem mass spectrometric sequencing with an automated MS-toMS/MS switching protocol.

The database search was performed with MASCOT Deamon (Matrix Science, London, UK) (Eng et al., 1994; Mann and Wilm, 1994; Pappin et al., 1993; Perkins et al., 1999). The generated pkl files were submitted to SWISS-PROT (release SwissProt_2012_01) and NCBInr (NCBInr_20120226).

\section{Solubility analysis of $\alpha$-casein}

Solubility of $\alpha$-casein, a representative protein that consists of multiple spots with different mobility due to the differences in its phosphorylation pattern in 2D-gels, was examined in each fixative and also in the solvents of the methacarn solution. One sample each was analyzed for comparison between fixatives or solvents of the methacarn solution. Five $\mathrm{mg}$ of $\alpha$-casein ( $\alpha$-casein from bovine milk; Sigma-Aldrich Co., St. Louis, MO, USA) was dissolved in $500 \mu \mathrm{l}$ of $50 \mathrm{mM}$ ammonium bicarbonate. The dissolved $\alpha$-casein $(1 \mathrm{mg}$ ) solution was dried, mixed either with $600 \mu \mathrm{l}$ of methacarn solution, methanol and chloroform mixture ( $6: 3)$, acetone, chloroform, or methanol, then subjected to extraction for $5 \mathrm{hr}$ at $4^{\circ} \mathrm{C}$. After centrifugation at $20,000 \times \mathrm{g}$ for $2 \mathrm{hr}$ at $4^{\circ} \mathrm{C}$, the dissolved $\alpha$-casein in the supernatant was measured by the weight of residual mass of the dried supernatant. The dissolved and non-dissolved $\alpha$-casein pellets were dissolved in lysis buffer and subjected to 2D-DIGE analysis.

\section{Statistical analysis}

Comparison of RIN between the unfixed tissue samples of the cingulate cortex and the cerebellar vermis were performed with the Student's $t$-test (homogeneity) or Aspin-Welch's $t$-test (heterogeneity) after analyses for homogeneity of variance using the F-test. Comparison of RIN among brain regions in each fixation or among unfixed and each fixed-tissue samples of the cinglate cortex and the cerebellar vermis were performed with the Tukey's (homogeneous) or Steel-Dwass's test (heterogeneous) after analyses for homogeneity of variance using Bartlett's test. Spot volume in 2D-DIGE analysis was compared between unfixed and each fixed-tissue samples for each matched spot and a Student's $t$-test or ANOVA test was applied using the triplicate values from each experimental condition.

\section{RESULTS}

\section{Integrity of total RNAs}

RIN was estimated in each region of the unfixed brain tissues (cingulate cortex and cerebellar vermis) and methacarn- or acetone-fixed tissues (cingulate cortex, cerebellar vermis, hippocampal dentate gyrus and corpus callosum). In the unfixed tissues, RIN in the cerebellar vermis was higher than that in the cingulate cortex at both PND 63 and PND 21 (Fig. 1A). In the methacarn-fixed tissues, RIN was similar among brain regions at both PND 63 and PND 21. In the acetone-fixed tissues, RIN of the cerebellar vermis was statistically higher as compared with the cingulate cortex or corpus callosum at PND 63. On PND 21 , RIN of the cerebellar vermis was statistically higher as compared with the corpus callosum.

Average of RINs of total RNAs in the cingulate cortex was similar between unfixed and methacarn-fixed tissues at PND 63, but it was significantly higher in unfixed tissues than in methacarn-fixed tissues at PND 21 
Methacarn for global expression analysis

A
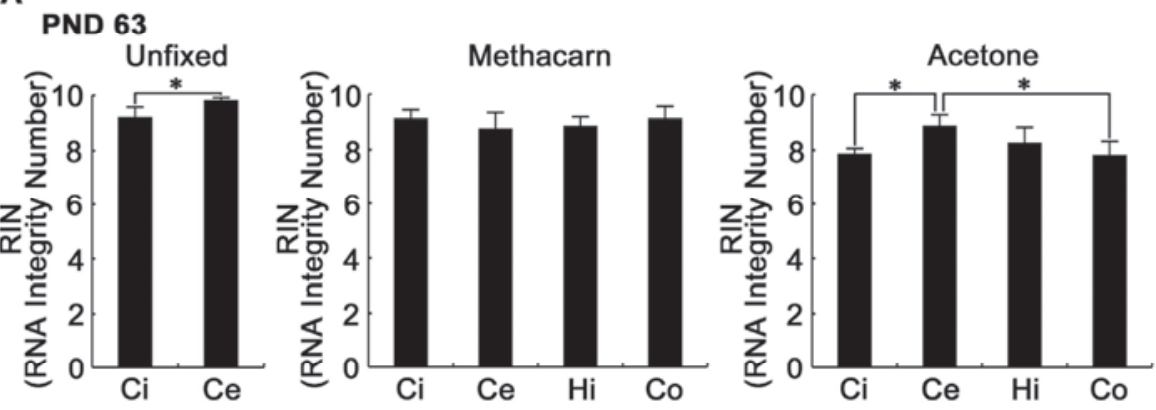

PND 21
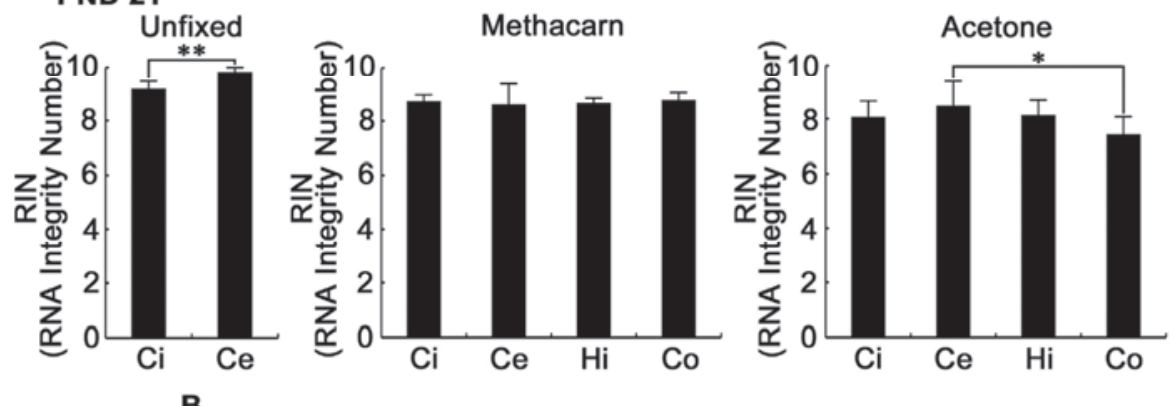

B $\mathrm{Ci}$
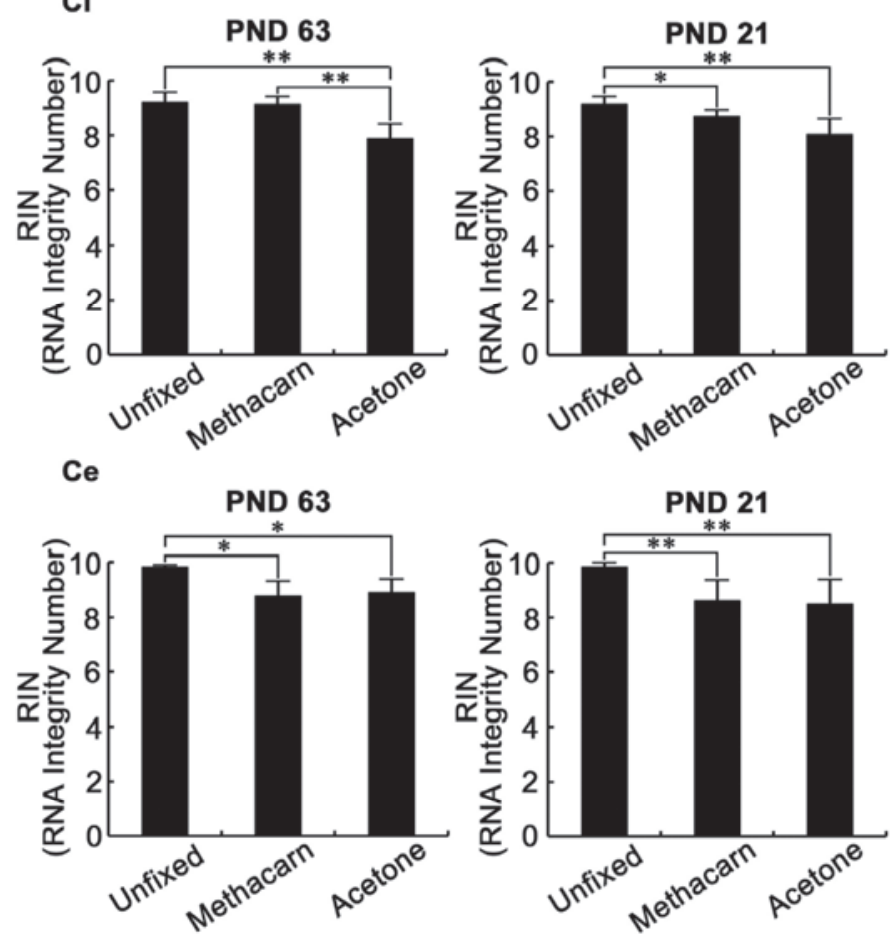

Fig. 1. RIN of total RNAs extracted from unfixed tissue, methacarn-fixed and acetone-fixed tissues. A. RIN values at each brain region. Data are expressed as mean + S.D. Number of animals is 4 at PND 63 and 8 at PND 21 in each tissue preparation. $*, * *$ : Significantly different in each comparison $(* p<0.05, * * p<0.01)$. Abbreviations: Ci, cingulate cortex; Ce, cerebellar vermis; Hi, hippocampal dentate gyrus; Co, corpus callosum. B. Average RIN values of the cingulate cortex and cerebellar vermis. Data are expressed as mean + S.D. Number of animals is 4 at PND 63 and 8 at PND 21 in each tissue preparation. *, **: Significantly different in each comparison $(* p<0.05, * * p<0.01)$. 


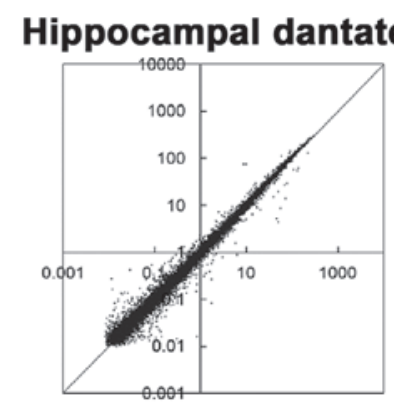

Methacarn

\section{Cingulate cortex}

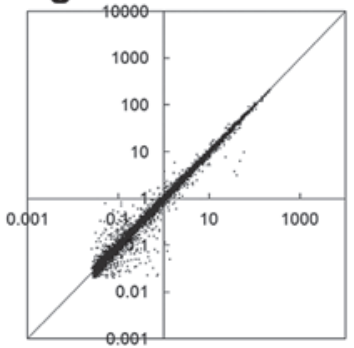

Methacarn

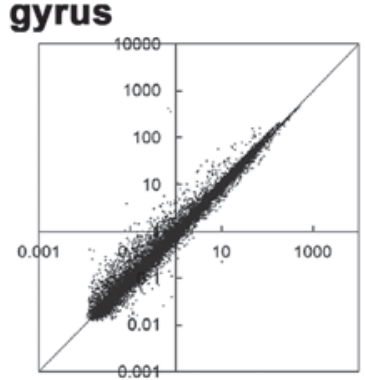

Acetone

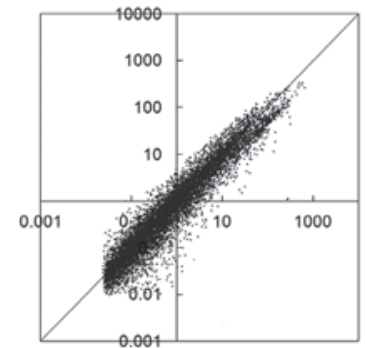

Acetone

\section{Corpus callosum}

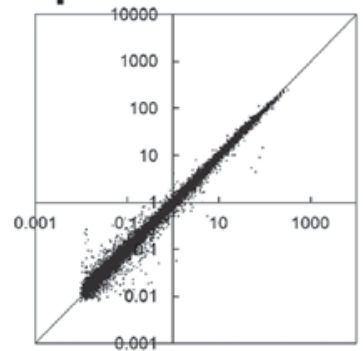

Methacarn

\section{Cerebellar vermis}

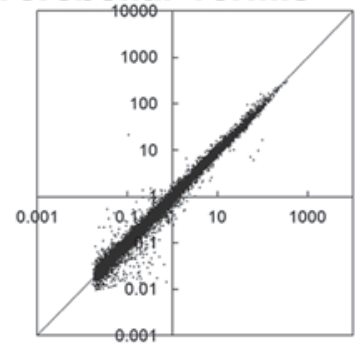

Methacarn

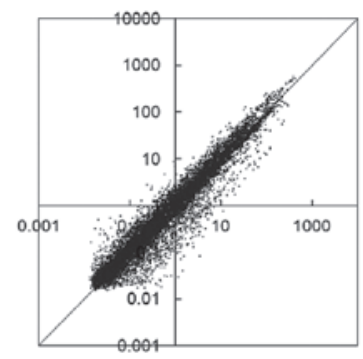

Acetone

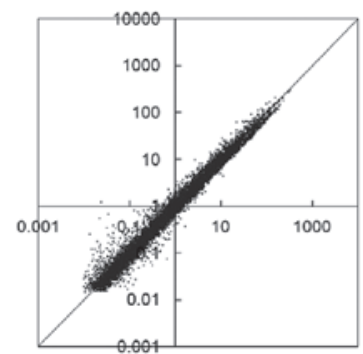

Acetone

Fig. 2. Scatter plot of individual gene expression within each fixative in four brain regions at PND 63. Three animal samples were analyzed in each brain region after fixation with methacarn or acetone.

(Fig. 1B). Average of RINs of unfixed cingulate cortex tissues was significantly higher than that of acetone-fixed tissues at both PND 63 and PND 21. Average of RINs of methacarn-fixed cingulate cortex tissues was significantly higher than that of acetone-fixed ones at PND 63, while it was unchanged between methacarn- and acetone-fixed cingulate cortex tissues at PND 21. Average of RINs of total RNAs in the cerebellar vermis was higher in unfixed tissue than in methacarn- or acetone-fixed tissues at both PND 63 and PND 21. Average of RINs was unchanged between methacarn- and acetone-fixed cerebellar vermis tissues at both PND 63 and PND 21.

\section{Variability in microarray data}

Figure 2 shows representative scatter plots between microarray gene expression data of each animal within the same fixative in four brain regions at PND 63. Higher correlations were observed in mRNA expression data in the methacarn-fixed tissues as compared with acetonefixed tissues in all brain regions. Table 1 shows average of coefficients of determination and their coefficients of variation in each brain region of methacarn- or acetonefixed tissues at PND 63 and PND 21. When these values were compared between fixatives, methacarn-fixed tissues showed higher coefficients of determination in each brain region as compared with acetone-fixed ones. Also, coefficients of variation were consistently lower in the methacarn-fixed tissues as compared with acetone-fixed ones.

\section{Integrity of polypeptides}

The visual pattern of resolved polypeptide bands and their intensities in polyacrylamide gels were similar between unfixed tissue, methacarn-fixed and acetonefixed tissues in the cingulate cortex and cerebellar vermis as examined at PND 63 (Fig. 3).

\section{Protein expression analysis by 2D-DIGE}

The visual patterns of polypeptide spots resolved in polyacrylamide gels did not differ between unfixed tissues and methacarn-fixed or acetone-fixed tissues in the cingulate cortex and cerebellar vermis as examined at PND 63 (Fig. 4). Approximately, 2,100-2,300 polypeptides were resolved in the 2D-gel. Almost all of the polypeptide spots showed no differences in spot volume and mobility in the 2D-gel between fixed and unfixed tissue samples. Standardized abundance of each polypeptide in the unfixed frozen, methacarn-fixed, or acetonefixed tissue was estimated by calculating the ratio of the 
Methacarn for global expression analysis

Table 1. Mean value of coefficients of determination and it's coefficients of variation in the scatter plot analysis of gene expressions in methacarn and acetone-fixed tissues

\begin{tabular}{|c|c|c|c|c|c|}
\hline & \multirow{3}{*}{$\begin{array}{c}\text { No. of } \\
\text { samples }\end{array}$} & \multicolumn{4}{|c|}{ Fixative } \\
\hline & & \multicolumn{2}{|c|}{ Methacarn } & \multicolumn{2}{|c|}{ Acetone } \\
\hline & & C.D. & C.V. & C.D. & C.V. \\
\hline \multicolumn{6}{|l|}{ PND 63 (Experiment 1) } \\
\hline Hippocampal dentate gyrus & 3 & 0.982 & 0.006 & 0.948 & 0.021 \\
\hline Corpus callosum & 3 & 0.985 & 0.005 & 0.982 & 0.006 \\
\hline Cingulate cortex & 3 & 0.993 & 0.001 & 0.883 & 0.110 \\
\hline Cerebellar vermis & 3 & 0.976 & 0.007 & 0.963 & 0.026 \\
\hline \multicolumn{6}{|l|}{ PND 21 (Experiment 2) } \\
\hline Hippocampal dentate gyrus & 3 & 0.978 & 0.012 & 0.977 & 0.010 \\
\hline Corpus callosum & 3 & 0.988 & 0.002 & 0.971 & 0.020 \\
\hline Cingulate cortex & 3 & 0.995 & 0.001 & 0.981 & 0.005 \\
\hline Cerebellar vermis & 3 & 0.990 & 0.005 & 0.923 & 0.061 \\
\hline
\end{tabular}

Abbreviations: C.D., coefficient of determination; C.V., coefficient of variation; PND, postnatal day.

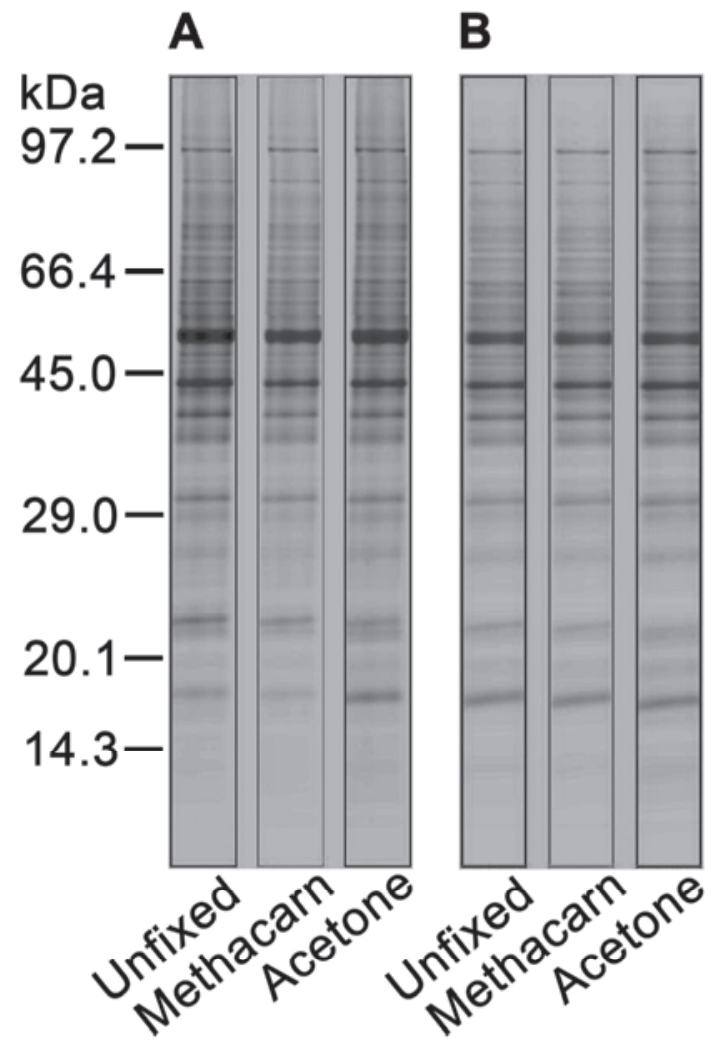

Fig. 3. Integrity of polypeptide bands in protein extracts from unfixed tissues, methacarn-fixed or acetone-fixed tissues at PND 63. A. Cingulate cortex. B. Cerebellar vermis. spot volume in unfixed frozen, or methacarn- or acetone-fixed samples to the volumes of pooled samples of all unfixed and fixed tissues. Average log ratios of standardized abundance in fixed samples per unfixed sample were then calculated. In the cingulate cortex, statistically significant differences in the standardized abundance were detected in 7 polypeptides in methacarn-fixed tissues and in 10 polypeptides in acetone-fixed tissues as compared with unfixed tissues (Table 2). In the cerebellar vermis, statistically significant differences in the standardized abundance were detected in 1 polypeptide in methacarn-fixed tissues and in 3 polypeptides in acetone-fixed tissues as compared with unfixed tissues (Table 3). All these polypeptides were $>29 \mathrm{kDa}$ in molecular weight. In both the cingulate cortex and cerebellar vermis, magnitudes of fluctuations in the standardized abundance in methacarn-fixed tissues were mostly smaller than those of acetone-fixed tissue. Among these polypeptides, both phosphoglycerate mutase 1 and triosephosphate isomerase were found to consist of three spots by MS analysis (Fig. 5). Reductions in spot volume of one of three separate spots by different isoelectric points were observed in acetone-fixed tissues as compared to that in unfixed tissues (Figs. 5 and 6). On the other hand, in methacarn-fixed tissues, three separate spot volumes were equivalent to those in unfixed tissues.

\section{Protein solubility in fixatives}

Solubility of $\alpha$-casein in each fixative and in solvents contained in the methacarn solution was examined (Table 4). 

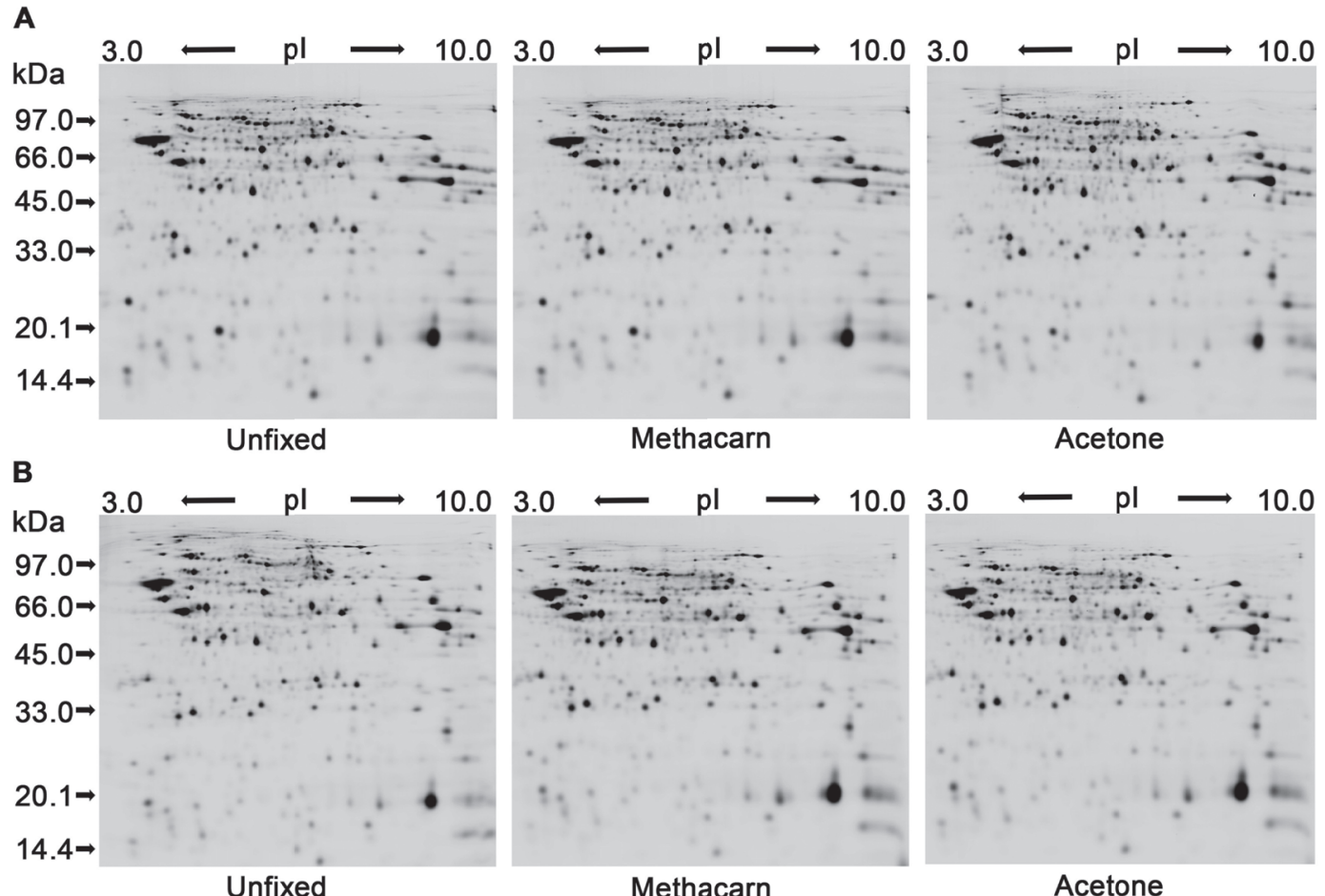

Methacarn

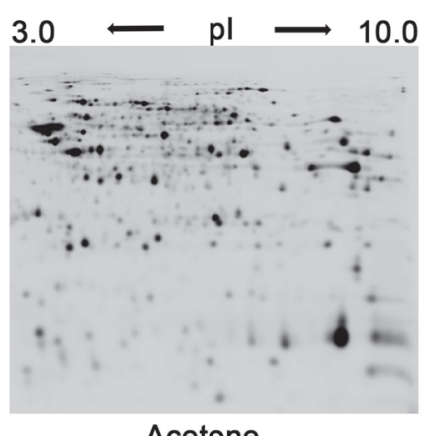

Fig. 4. 2D-gel image of polypeptides extracted from unfixed tissues and methacarn-fixed or acetone-fixed tissues at PND 63. A. Cingulate cortex. B. Cerebellar vermis.

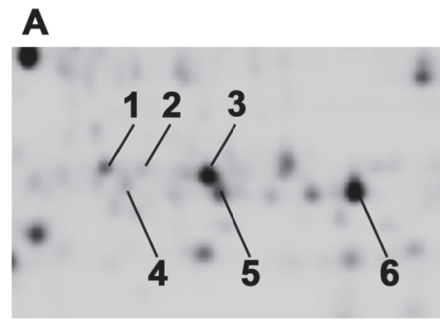

Unfixed

B

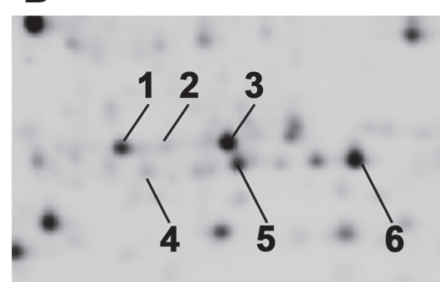

Unfixed

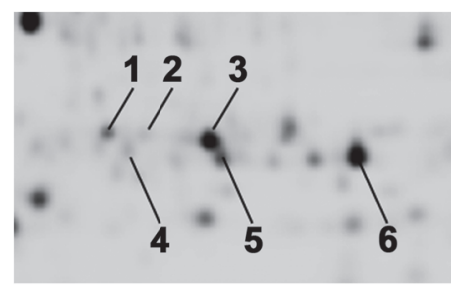

Methacarn

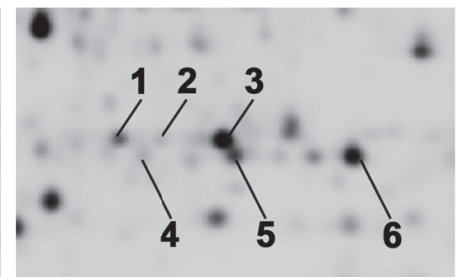

Methacarn

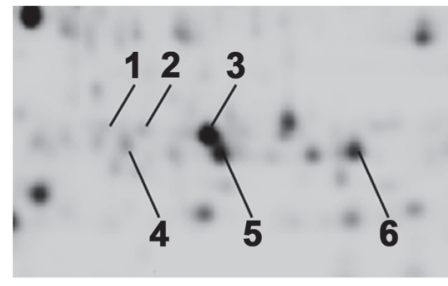

Acetone

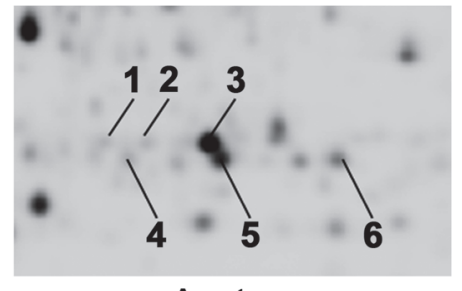

Acetone

Fig. 5. 2D-gel image of polypeptides extracted from unfixed tissues and methacarn-fixed or acetone-fixed tissues at PND 63. Representative spots of phosphoglycerate mutase 1 (spot No. 1-3) and triosephosphate isomerase (spot No. 4-6), each consisting of three spots. Reductions of spot volume of one of three spots (spot No. 1 of phosphoglycerate mutase 1 and spot No.6 of triosephosphate isomerase) are evident in acetone-fixed tissue as compared to that in unfixed tissue. A. Cingulate cortex. B. Cerebellar vermis. 
Methacarn for global expression analysis

Table 2. Average ratio of standardized abundance of polypeptides in methacarn or acetone-fixed tissue to that of unfixed tissue from the cingulate cortex

\begin{tabular}{ccll}
\hline \multicolumn{2}{c}{ Average ratio of standardized abundance } & \\
\cline { 1 - 2 } Methacarn / Unfixed & Acetone / Unfixed & & Protein \\
\hline $1.82^{\text {a }}$ & $2.32^{*}$ & & Heat shock cognate 71 kDa protein \\
$2.35^{*}$ & $3.17^{*}$ & & Undetermined polypeptide \#1 \\
$-2.29^{*}$ & -1.40 & & Serum albumin \\
1.51 & $2.62^{*}$ & & Dihydropyrimidinase-related protein 2 \\
1.71 & $2.16^{*}$ & & Undetermined polypeptide \#2 \\
$2.44^{*}$ & $3.73^{*}$ & Actin-related protein 3 \\
$1.70^{*}$ & $2.79^{*}$ & Alpha-enolase \\
$1.74^{*}$ & $2.27^{*}$ & & Undetermined polypeptide \#3 \\
$2.16^{*}$ & 1.03 & Eukaryotic translation initiation factor 3 subunit I \\
1.40 & $2.04^{*}$ & & NAD-dependent deacetylase sirtuin-2 \\
$-1.54 *$ & $-5.20^{*}$ & Phosphoglycerate mutase 1 \\
1.09 & $-2.05^{*}$ & Triosephosphate isomerase \\
\hline
\end{tabular}

aStandardized abundance of each polypeptide was estimated by measuring the ratio of spot volume in each of unfixed frozen, or methacarn- or acetone-fixed samples to the spot volume of pooled samples of all unfixed and fixed tissues. Average log ratio of standardized abundance in fixed samples per unfixed sample was then calculated. Number of animals examined is 4 in each tissue preparation. *Significantly different between methacarn or acetone-fixed tissue samples and unfixed tissue samples $(p<0.01)$.

Table 3. Average ratio of standardized abundance of polypeptides in methacarn or acetone-fixed tissue to that of unfixed tissue from the cerebellar vermis

\begin{tabular}{ccll}
\hline \multicolumn{2}{l}{ Average ratio of standardized abundance } & \\
\cline { 1 - 2 } Methacarn / Unfixed & Acetone / Unfixed & Protein \\
\hline $2.00^{\text {a }}$ & $3.56^{*}$ & & Actin-related protein 3 \\
$-1.48^{*}$ & $-4.11^{*}$ & & Phosphoglycerate mutase 1 \\
-1.29 & $-2.42^{*}$ & Hypoxanthine-guanine phosphoribosyltransferase \\
1.10 & $-2.20^{*}$ & Triosephosphate isomerase \\
\hline
\end{tabular}

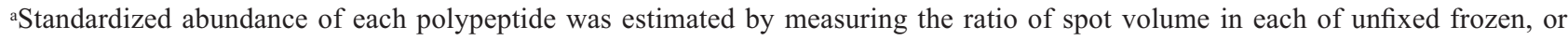
methacarn- or acetone-fixed samples to the spot volume of pooled samples of all unfixed and fixed tissues. Average log ratio of standardized abundance in fixed samples per unfixed sample was then calculated. Number of animals examined is 4 in each tissue preparation. *Significantly different between methacarn or acetone-fixed tissue samples and unfixed tissue samples $(p<0.01)$.

The ratio of solubility to total input was much lower in methacarn solution than in acetone or in the solvents of the methacarn solution. In 2D-DIGE analysis, we selected six separate polypeptide spots of $\alpha$-casein by different isoelectric points. There was one spot with reduced spot volume in acetone (Spot No. 3); however, there were no spots showing variability in solubility in methacarn solution (Figs. 7 and 8).

\section{DISCUSSION}

There are two critical points for global analysis of macromolecules using fixed-tissues, i.e., i) integrity of extract-
Table 4. Solubility of $\alpha$-casein to fixatives or solvents consisting of methacarn solution

\begin{tabular}{lc}
\hline Solvent & Solubility of $\alpha$-casein (\%) \\
\hline Methacarn & $0.2^{\mathrm{a}}$ \\
Acetone & 8.7 \\
Methanol:chloroform $(6: 3)$ & 10.1 \\
Methanol & 5.2 \\
Chloroform & 9.5
\end{tabular}

${ }^{a}$ Number of examination is one. 

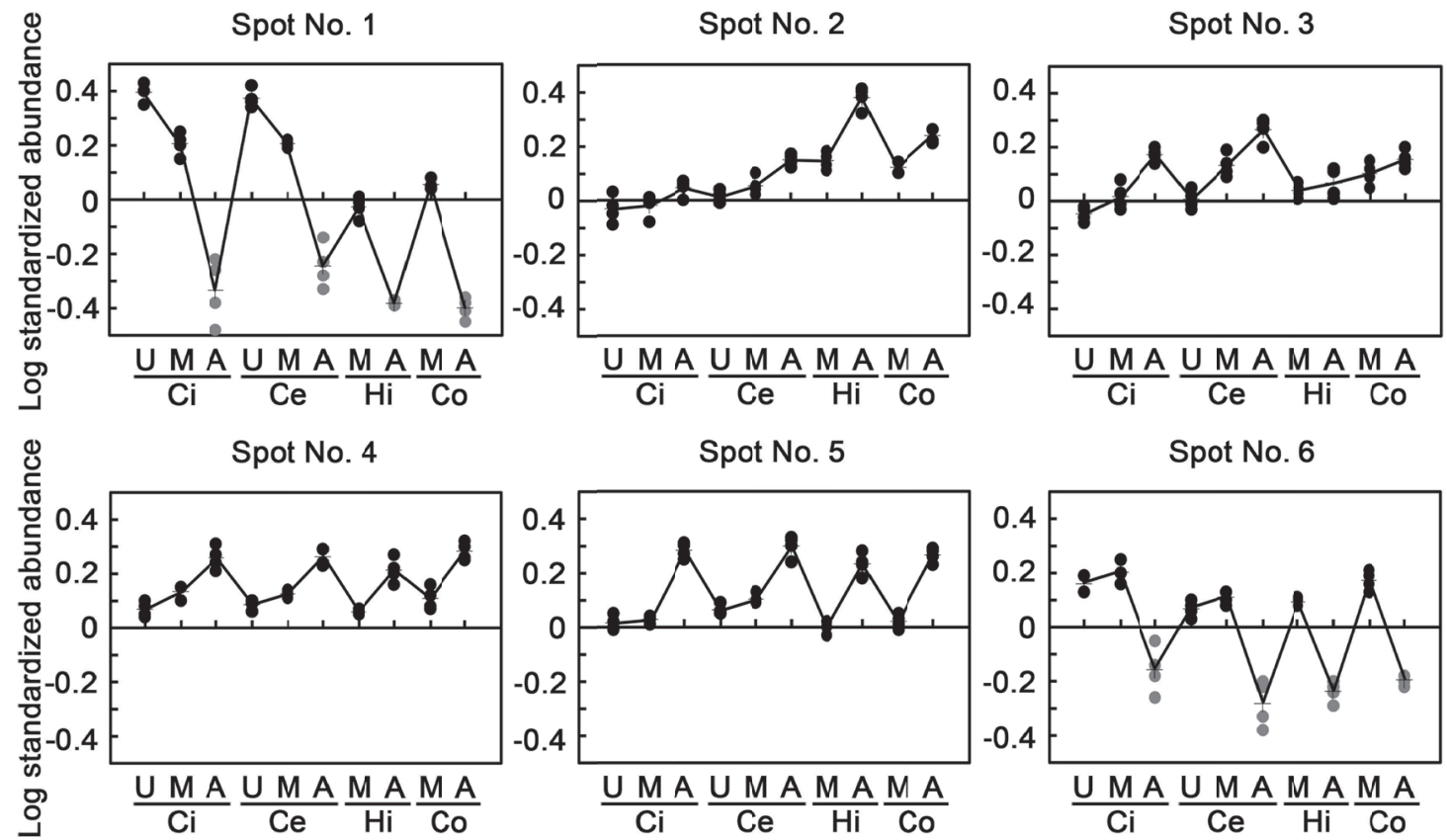

Fig. 6. Log standardized abundance of each six spots of phosphoglycerate mutase 1 (spot No. 1-3) and triosephosphate isomerase (spot No. 4-6) shown in Fig. 5. Number of animals is 4 for each tissue preparation. In acetone-fixed tissue, log standardized abundance of spot 1 of phosphoglycerate mutase 1 and spot 6 of triosephosphate isomerase are decreased as compared to that in unfixed tissue. In methacarn-fixed tissue, three spot volumes of each protein are equivalent to unfixed tissue. Abbreviations: $\mathrm{U}$, unfixed tissue; $\mathrm{M}$, methacarn-fixed tissue; A, acetone-fixed tissue; $\mathrm{Ci}$, cingulate cortex; $\mathrm{Ce}$, cerebellar vermis; Hi, hippocampal dentate gyrus; Co, corpus callosum.

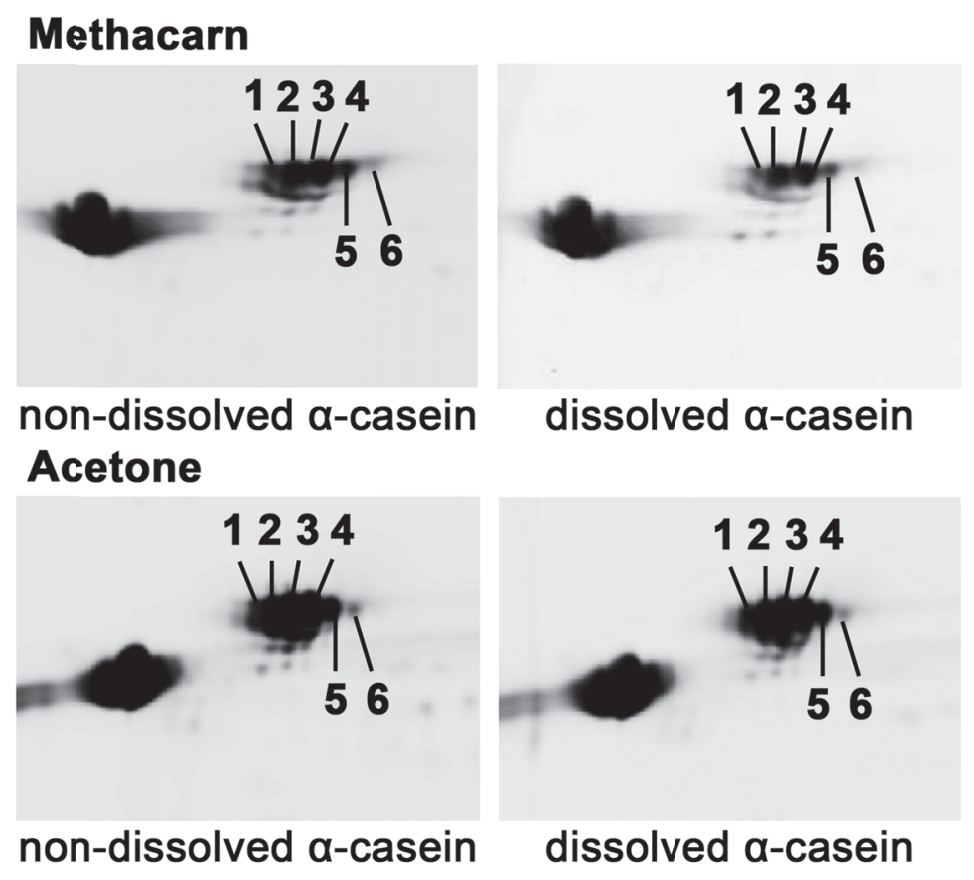

Fig. 7. 2D-gel image of dissolved and non-dissolved $\alpha$-casein to methacarn solution or acetone. 

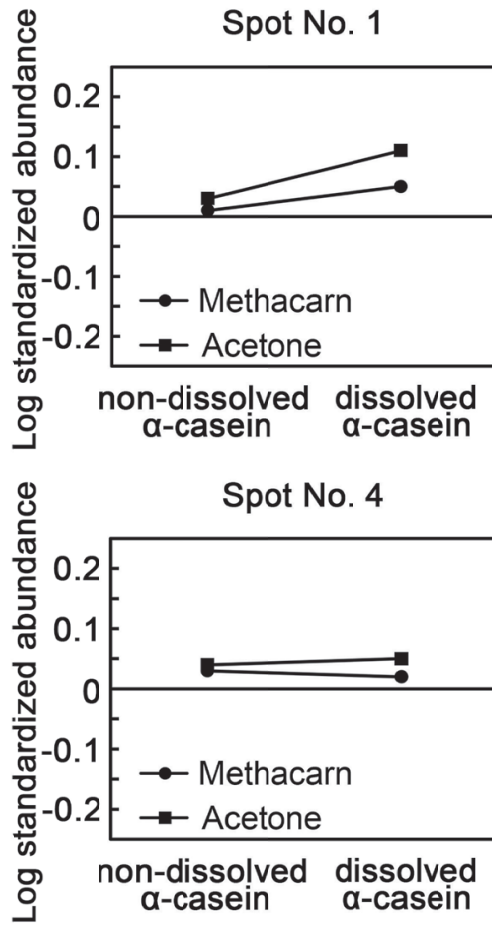

Spot No. 2

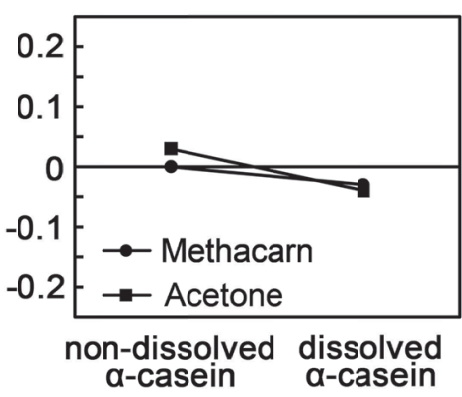

Spot No. 5

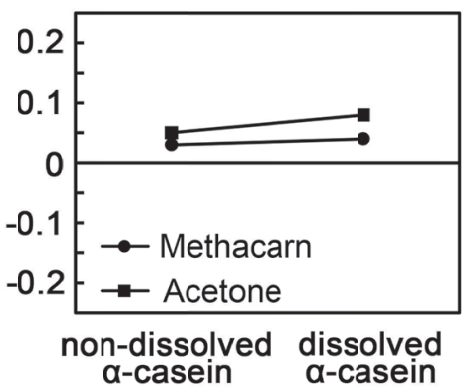

Spot No. 3

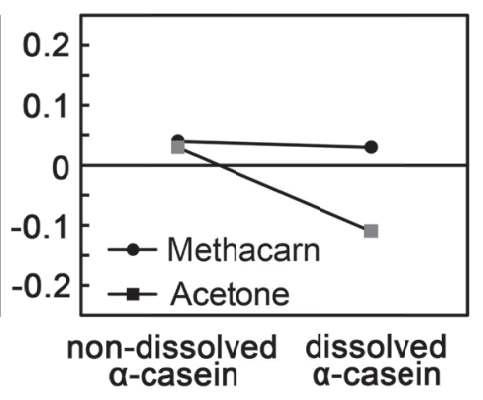

Spot No. 6

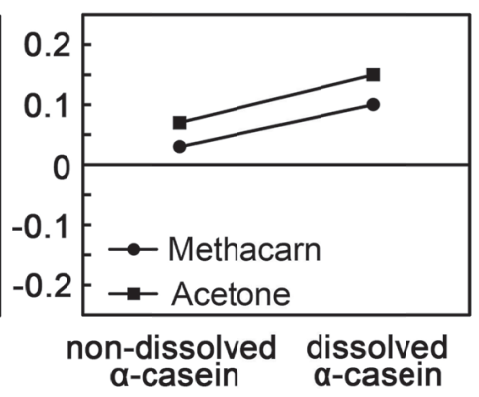

Fig. 8. Log standardized abundance of each six spots of $\alpha$-casein shown in Fig. 7. Number of sample pair is one. By extraction with acetone, $\log$ standardized abundance of the spot 3 of dissolved $\alpha$-casein is decreased as compared to that of non-dissolved $\alpha$-casein. By extraction with methacarn solution, there were no spots showing variability in the solubility.

ed molecules (Finke et al., 1993; Stanta and Schneider, 1991); and ii) fidelity in expression changes as compared with unfixed tissues. In the present study, we found that the integrity of total RNAs by methacarn fixation was superior to acetone fixation and next to the unfixation. Extracted polypeptides resolved in SDS-PAGE showed similar integrity between unfixed tissue and methacarn or acetone fixation. When the fidelity of mRNA expression changes in microarray analysis was compared, methacarn fixation resulted in lower expression variability between samples than acetone fixation. With regard to the polypeptide expression, fidelity was mostly equivalent between the two fixation methods in 2D-DIGE, while extracting effect of polypeptides may occur during fixation with acetone in relation with their phosphorylation status.

In contrast to the very low performance on extraction of total RNA with formaldehyde-based fixatives, irrespective of temperature control by fixation at $4{ }^{\circ} \mathrm{C}$ (Bussolati et al., 2011; Farragher et al., 2008), organic solventbased protein-precipitating fixatives usually give high yields and integrity of extracted total RNA (Goldsworthy et al., 1999; Shibutani et al., 2000; Srinivasan et al.,
2002). For the purpose of microarray analysis, the lower limit of RIN required should be higher than 7.0 (Imbeaud et al., 2005; Thompson et al., 2007). In the present study, mean RINs of extracted total RNA from methacarn- or acetone-fixed tissues were higher than 8.0, and methacarn resulted in superior RIN compared with acetone. Importantly, RINs were constant in all anatomical locations sampled from methacarn-fixed tissues but variability was seen in RIN values between the brain regions in acetone-fixed tissues, suggesting that whole brain fixation using methacarn is superior to acetone for comparison of gene expression changes between anatomically different regions within the brain. While the reason for the difference in RIN values between tissue regions by acetone fixation was unclear, differences in the RIN value between tissue regions was unrelated to the distance of tissue from the brain surface as an interface with fixative, suggesting that speed of tissue penetration of acetone during fixation may not be responsible for this difference.

Previously, scatter plots of mRNA expression microarray data between frozen tissues and $70 \%$ ethanol- or formalin-fixed paraffin-embedded tissues showed low corre- 


\section{H. Akane et al.}

lation (Cox et al., 2008). This result might be due to low integrity of total RNA caused by these fixatives in combination with paraffin-embedding. On the other hand, we have already shown that methacarn-fixed tissue samples, even after paraffin-embedding, show a high fidelity in global expression changes in microarray analysis with unfixed tissues (Shibutani et al., 2007). In the present study, when methacarn and acetone were compared in terms of the global expression changes by microarrays, higher correlations in scatter plot were observed in the methacarn-fixed tissues in all brain regions of both PND 21 and PND 63, while we could not compare expression changes with unfixed tissue samples due to the inaccuracy in the sampling of anatomically-specific regions in unfixed tissue. Higher integrity of extracted total RNAs may be responsible for the lower inter-animal variability in the expression with methacarn as compared with acetone. For gene expression analysis in anatomically-specific regions, application of microdissection technique for tissue sampling may be recommended (Böhm et al., 2005). However, it is laborious and time-consuming for collection of tissue samples as we examined previously (Fujimoto et al., 2012; Saegusa et al., 2010; Shibutani et al., 2007; Woo et al., 2009). Of note, low variability in mRNA expression between samples could be achieved even with manual dissection using punch-biopsy devices for tissue sampling in the present study. If a tissue area dissected by punch-biopsy devices fulfills the experimental purpose of analysis, the manual dissection technique used here in combination with methacarn fixation may provide a high throughput data collection in mRNA expression with low variability between samples.

In formalin-fixed tissue samples, reduction in spot signal intensity and acidic shift of extracted polypeptides usually occur in 2D-DIGE analysis (Tanca et al., 2011). Also, microdissected paraffin-embedded tissue samples stained with hematoxylin and eosin after fixation with $70 \%$ ethanol or acetone resulted in reduction of spot number of proteins in 2D-gel (De Souza et al., 2004). In the present study, the visual pattern of resolved polypeptide bands and their intensities in polyacrylamide gels were similar between unfixed tissues and methacarn- or acetone-fixed tissues, thus both fixatives have not affected the quality of polypeptides. In analysis of 2D-DIGE, almost all of the polypeptides resolved on gel, approximately 2,100-2,300 in number, were unchanged in their spot volumes and mobility between fixed and unfixed tissues, suggesting that proteome analysis could be applied even after tissue fixation with protein-precipitating agents. Interestingly, although a small number of polypeptides showed different expression levels between unfixed tis- sues and fixed tissues, methacarn was superior to acetone in the number of polypeptides affected. In addition, expression level of one of three separated spots of two polypeptides by the difference in the posttranslational modification was affected by acetone fixation, while it was unaffected by methacarn fixation.

With regard to possibility of polypeptide extraction during fixation, we found $\alpha$-casein, which is a representative phosphorylated protein, was scarcely soluble in methacarn solution in the present study, while it was slightly soluble in acetone. The solubility in acetone differed in one of six spots showing different phosphorylation patterns. These results suggest that expression levels of polypeptides extracted from acetone-fixed tissues may be affected according to their phosphorylation status. On the other hand, we previously found some unknown modifications on certain population of polypeptides to affect the mobility in SDS-PAGE in methacarn-fixed paraffinembedded sections in western blot analysis (Shibutani et al., 2000). Because we did not find mobility shift of molecules in almost all proteins in the present study, the mobility shift observed in our previous study was due to some modification during paraffin-embedding.

In conclusions, whole brain fixation of rats using methacarn in combination with manual dissection using punch-biopsy devices enabled us to extract total RNAs and polypeptides with high integrities and expression fidelity sufficient for application to global expression analysis of both mRNAs and polypeptides. Extracted total RNA samples were found to show uniform integrity between brain regions, suggestive of methacarn fixation as a fast and effective method to preserve RNA within fixed tissues. Extraction of polypeptides during fixation was found to be negligible with methacarn. Thus, whole brain fixation with methacarn is judged to be well suited for high throughput analysis of gene expression in anatomically-specific regions in the framework of toxicity testing in rodents.

\section{ACKNOWLEDGMENTS}

This work was supported by a grant from Ministry of Economy, Trade and Industry (METI), Japan. All authors disclose that there are no conflicts of interest that could inappropriately influence the outcome of this study.

\section{REFERENCES}

Banks, P.M. (1979): Diagnostic applications of an immunoperoxidase method in hematopathology. J. Histochem. Cytochem., 27, 1192-1194.

Böhm, C., Newrzella, D. and Sorgenfrei, O. (2005): Laser microdis- 
Methacarn for global expression analysis

section in CNS research. Drug Discov. Today, 10, 1167-1174.

Bussolati, G., Annaratone, L., Medico, E., D’Armento, G. and Sapino, A. (2011): Formalin fixation at low temperature better preserves nucleic acid integrity. PLoS One, 6, e21043.

Cox, M.L., Eddy, S.M., Stewart, Z.S., Kennel, M.R., Man, M.Z., Paulauskis, J.D. and Dunstan, R.W. (2008): Investigating fixative-induced changes in RNA quality and utility by microarray analysis. Exp. Mol. Pathol., 84, 156-172.

Delfour, C., Roger, P., Bret, C., Berthe, M.L., Rochaix, P., Kalfa, N., Raynaud, P., Bibeau, F., Maudelonde, T. and Boulle, N. (2006): RCL2, a new fixative, preserves morphology and nucleic acid integrity in paraffin-embedded breast carcinoma and microdissected breast tumor cells. J. Mol. Diagn., 8, 157-169.

De Souza, A.I., McGregor, E., Dunn, M.J. and Rose, M.L. (2004): Preparation of human heart for laser microdissection and proteomics. Proteomics, 4, 578-586.

Eng, J.K., McCormack, A.L. and Yates, J.R.3rd. (1994): An approach to correlate tandem mass spectral data of peptides with amino acid sequences in a protein database. J. Am. Soc. Mass Spectrom., 11, 976-989.

Farragher, S.M., Tanney, A., Kennedy, R.D. and Paul Harkin, D. (2008): RNA expression analysis from formalin fixed paraffin embedded tissues. Histochem. Cell Biol., 130, 435-445.

Finke, J., Fritzen, R., Ternes, P., Lange, W. and Dölken, G. (1993): An improved strategy and a useful housekeeping gene for RNA analysis from formalin-fixed, paraffin-embedded tissues by PCR. Biotechniques, 14, 448-453.

Fujimoto, H., Woo, G.H., Inoue, K., Igarashi, K., Kanno, J., Hirose, M., Nishikawa, A. and Shibutani, M. (2012): Increased cellular distribution of vimentin and Ret in the cingulum induced by developmental hypothyroidism in rat offspring maternally exposed to anti-thyroid agents. Reprod. Toxicol., 34, 93-100.

Goldsworthy, S.M., Stockton, P.S., Trempus, C.S., Foley, J.F. and Maronpot, R.R. (1999): Effects of fixation on RNA extraction and amplification from laser capture microdissected tissue. Mol. Carcinog., 25, 86-91.

Heijne, W.H., Kienhuis, A.S., van Ommen, B., Stierum, R.H. and Groten, J.P. (2005): Systems toxicology: applications of toxicogenomics, transcriptomics, proteomics and metabolomics in toxicology. Expert Rev. Proteomics, 5, 767-780.

Imbeaud, S., Graudens, E., Boulanger, V., Barlet, X., Zaborski, P., Eveno, E., Mueller, O., Schroeder, A. and Auffray, C. (2005): Towards standardization of RNA quality assessment using userindependent classifiers of microcapillary electrophoresis traces. Nucleic Acids Res., 33, e56.

Mann, M. and Wilm, M. (1994): Error-tolerant identification of peptides in sequence databases by peptide sequence tags. Anal. Chem., 66, 4390-4399.

Mitchell, D., Ibrahim, S. and Gusterson, B.A. (1985): Improved immunohistochemical localization of tissue antigens using modified methacarn fixation. J. Histochem. Cytochem., 33, 491-495.

Ornstein, D.K., Gillespie, J.W., Paweletz, C.P., Duray, P.H., Herring, J., Vocke, C.D., Topalian, S.L., Bostwick, D.G., Linehan, W.M., Petricoin, E.F. $3^{\text {rd }}$ and Emmert-Buck, M.R. (2000): Proteomic analysis of laser capture microdissected human prostate cancer and in vitro prostate cell lines. Electrophoresis, 21, 2235-2242.

Orstavik, T.B., Brandtzaeg, P., Nustad, K. and Pierce, J.V. (1981): Effects of different tissue processing methods on the immunohistochemical localization of kallikrein in the pancreas. J. Histochem. Cytochem., 29, 985-988.

Pappin, D.J., Hojrup, P. and Bleasby, A.J. (1993): Rapid identification of proteins by peptide-mass fingerprinting. Curr. Biol., $\mathbf{3}$, 327-332.
Perkins, D.N., Pappin, D.J., Creasy, D.M. and Cottrell, J.S. (1999): Probability-based protein identification by searching sequence databases using mass spectrometry data. Electrophoresis, 20, 3551-3567.

Puchtler, H., Waldrop, F.S., Meloan, S.N., Terry, M.S. and Conner, H.M. (1970): Methacarn (methanol-Carnoy) fixation. Practical and theoretical considerations. Histochemie, 21, 97-116.

Rognum, T.O., Brandtzaeg, P., Orjasaeter, H. and Fausa, O. (1980): Immunohistochemistry of epithelial cell markers in normal and pathological colon mucosa. Comparison of results based on routine formalin- and cold ethanol-fixation methods. Histochemistry, 67, 7-21.

Saegusa, Y., Woo, G.H., Fujimoto, H., Inoue, K., Takahashi, M., Hirose, M., Igarashi, K., Kanno, J., Mitsumori, K., Nishikawa, A. and Shibutani, M. (2010): Gene expression profiling and cellular distribution of molecules with altered expression in the hippocampal CA1 region after developmental exposure to anti-thyroid agents in rats. J. Vet. Med. Sci., 72, 187-195.

Shibutani, M., Lee, K.Y., Igarashi, K., Woo, G.H., Inoue, K., Nishimura, T. and Hirose, M. (2007): Hypothalamus region-specific global gene expression profiling in early stages of central endocrine disruption in rat neonates injected with estradiol benzoate or flutamide. Dev. Neurobiol., 67, 253-269.

Shibutani, M. and Uneyama, C. (2002): Methacarn: a fixation tool for multipurpose genetic analysis from paraffin-embedded tissues. Methods Enzymol., 356, 114-125.

Shibutani, M., Uneyama, C., Miyazaki, K., Toyoda, K. and Hirose, M. (2000): Methacarn fixation: a novel tool for analysis of gene expressions in paraffin-embedded tissue specimens. Lab. Invest., 80, 199-208.

Srinivasan, M., Sedmak, D. and Jewell, S. (2002): Effect of fixatives and tissue processing on the content and integrity of nucleic acids. Am. J. Pathol., 161, 1961-1971.

Stanta, G. and Schneider, C. (1991): RNA extracted from paraffinembedded human tissues is amenable to analysis by PCR amplification. Biotechniques, 11, 304-308.

Takagi, H., Shibutani, M., Kato, N., Fujita, H., Lee, K.Y., Takigami, S., Mitsumori, K. and Hirose, M. (2004): Microdissected region-specific gene expression analysis with methacarn-fixed, paraffin-embedded tissues by real-time RT-PCR. J. Histochem. Cytochem., 52, 903-913.

Tanca, A., Pagnozzi, D., Falchi, G., Tonelli, R., Rocca, S., Roggio, T., Uzzau, S. and Addis, M.F. (2011): Application of 2-D DIGE to formalin-fixed, paraffin-embedded tissues. Proteomics, 11, 1005-1011.

Thompson, K.L., Pine, P.S., Rosenzweig, B.A., Turpaz, Y. and Retief, J. (2007): Characterization of the effect of sample quality on high density oligonucleotide microarray data using progressively degraded rat liver RNA. BMC Biotechnol, 7, 57.

Uneyama, C., Shibutani, M., Masutomi, N., Takagi, H. and Hirose, M. (2002): Methacarn fixation for genomic DNA analysis in microdissected, paraffin-embedded tissue specimens. J. Histochem. Cytochem., 50, 1237-1245.

Woo, G.H., Takahashi, M., Inoue, K., Fujimoto, H., Igarashi, K., Kanno, J., Hirose, M., Nishikawa, A. and Shibutani, M. (2009): Cellular distributions of molecules with altered expression specific to thyroid proliferative lesions developing in a rat thyroid carcinogenesis model. Cancer Sci., 100, 617-625.

Yan, F., Wu, X., Crawford, M., Duan, W., Wilding, E.E., Gao, L., Nana-Sinkam, S.P., Villalona-Calero, M.A., Baiocchi, R.A. and Otterson, G.A. (2010): The search for an optimal DNA, RNA, and protein detection by in situ hybridization, immunohistochemistry, and solution-based methods. Methods, 52, 281-286. 